\section{ORIGINAL RESEARCH}

\author{
B. Alkonyi \\ C. Juhász \\ O. Muzik \\ M.E. Behen \\ J.-W. Jeong \\ H.T. Chugani
}

\title{
Thalamocortical Connectivity in Healthy Children: Asymmetries and Robust Developmental Changes between Ages 8 and 17 Years
}

\begin{abstract}
BACKGROUND AND PURPOSE: Thalamocortical connections play a crucial role in complex cognitive functioning, and several neuropsychiatric disorders may involve aberrant thalamocortical circuitry. Here, we quantified the cortical pattern and age-related changes of thalamocortical connections by using probabilistic tractography in children and adolescents. We hypothesized that detectable asymmetry (left>right) exists in thalamocortical fiber connections and the connectivity increases with age during maturation.
\end{abstract}

MATERIALS AND METHODS: Diffusion tensor imaging was acquired in 15 normally developing children (age range, 8.3-17.3 years; 11 males), and fiber tracking was initiated from the thalami. The cortical distribution of ipsilateral thalamocortical fibers was quantified by using a landmark-constrained conformal mapping technique. Furthermore, hemispheric asymmetries and potential age-related changes in regional thalamocortical connections were assessed.

RESULTS: The left thalamus had significantly higher overall cortical connectivity than the right thalamus $(P<.001)$. Left prefrontal cortical areas showed significantly higher thalamic connectivity compared with homotopic regions of the right hemisphere $(P<.001)$, regardless of the applied parameters. There was an increase of overall thalamocortical connectivity with age, with the most pronounced age-related increases in bilateral prefrontal areas $(P<.002)$. However, thalamic connectivity of some other cortical regions (right sensorimotor, left inferior temporal) showed a decrease with age.

CoNCLUSIONS: Our results indicate a region-specific left>right asymmetry and robust developmental changes in thalamocortical (particularly thalamo-prefrontal) connectivity during late childhood and adolescence. These data further add to our knowledge about structural lateralizations and their development in the maturing brain.

ABBREVIATIONS: $A D C=$ apparent diffusion coefficient; $A l=$ asymmetry index; $A S S E T=$ array spatial sensitivity encoding technique; DTI = diffusion tensor imaging; FA = fractional anisotropy; FSPGR = fast spoiled gradient recalled; FTE = finite target element; ICBM = International Consortium for Brain Mapping; ICC = intraclass correlation coefficient; PDF = probability density function; $\mathrm{ROI}=$ region of interest

T he thalamus is a major relay center of the brain with extensive, reciprocal cortical and subcortical connections. Thalamocortical pathways play a crucial role not only in transferring sensory inputs to the neocortex but also in higher cognitive functioning. ${ }^{1,2}$ Abnormalities of the cortico-(striato)thalamic circuit are involved in the pathophysiology of various neurologic and neuropsychiatric disorders. ${ }^{3,4}$

Previous functional MR imaging studies have enhanced our understanding of the network connections between the

Received March 31, 2010; accepted after revision September 29.

From the Carman and Ann Adams Department of Pediatrics (B.A., C.J., O.M., M.E.B., J.-W.J., H.T.C.) and Department of Neurology (C.J., O.M., M.E.B., H.T.C.), Wayne State University School of Medicine; and Translational Imaging Center (B.A., C.J., O.M., M.E.B., J.-W.J., H.T.C.), Children's Hospital of Michigan, Detroit, Michigan.

The present study was partially supported by National Institute of Neurological Disorders and Stroke (NINDS) grants R01 NS041922 (to C.J.) and R01 NS34488 (to H.T.C.). The project also was supported in part by National Institute of Mental Health (NIMH) grant K08 MH079176 (to M.E.B). The content is solely the responsibility of the authors and does not necessarily represent the official views of the NINDS or NIMH.

Please address correspondence to Csaba Juhász, MD, PhD, Department of Pediatrics and Neurology, Translational Imaging Center, Children's Hospital of Michigan, 3901 Beaubien Blvd, Detroit, Ml 48201; e-mail: juhasz@pet.wayne.edu

Indicates open access to non-subscribers at www.ajnr.org

DOI 10.3174/ajnr.A2417 thalamus and cortex. ${ }^{5,6}$ Results of the DTI-based segmentation fit well with prior knowledge of thalamocortical connections obtained from invasive tract-tracing studies in animal models. ${ }^{7}$ Moreover, functional MR imaging and DTI approaches have provided concordant results with respect to functional and connectivity-based segmentation of the thalamus. $^{5,8}$

Structural imaging studies have identified hemispheric asymmetries in the human brain. ${ }^{9-11}$ These anatomic asymmetries have been linked to the lateralization of motor, cognitive, and neurobehavioral functions, and their dynamic changes during childhood have been associated with development of cognitive skills. ${ }^{9,12,13}$ Numerous volumetry and DTI studies have reported age-related changes of cortical and subcortical gray matter densities, cortical thickness, and measures of white matter integrity in children, adolescents, and even in young adults. ${ }^{9,14-18}$ However, hemispheric differences in the cortical connectivity of the thalamus and potential age-related changes in the pattern of thalamocortical fibers have not been specifically addressed in children and may be key in understanding the altered, abnormal patterns seen in certain neurologic and neuropsychiatric disorders. ${ }^{3,4}$

DTI-based probabilistic tractography is a novel fiber tracking procedure that is suitable for the assessment and quantifi- 
cation of interregional white matter connectivity between gray matter structures such as the thalamus and the cerebral cortex. ${ }^{19}$ Its main advantage over deterministic algorithms is that it is capable of crossing low-anisotropy regions and thus, at least partially, identifying minor fiber tracts.

Here, we combined probabilistic DTI tractography with a landmark-based cortical surface parcellation and mapping technique implemented in each subject's native image space to assess the regional cortical patterns and age-related differences of thalamocortical connections in a sample of healthy children and adolescents. We hypothesized that more thalamocortical fiber tracts would be traced in the left (dominant) hemisphere. Furthermore, we hypothesized that the overall thalamocortical connectivity increases with age and that this increase may be more pronounced in certain segments of thalamocortical fibers.

\section{Materials and Methods}

\section{Subjects}

Fifteen healthy children and adolescents (mean age, 12.3 years; range, 8.3-17.3 years; 11 males; 14 right-handed, 1 ambidextrous) with no history of neurologic or psychological disorders or brain injury were involved in the present study. All children underwent intellectual and behavioral screening by using the Wechsler Intelligence Scales for Children-Third Edition ${ }^{20}$ and Behavioral Assessment System for Children-Second Edition, ${ }^{21}$ respectively, and a social-historical interview to ensure normal intellectual functioning and to rule out any current or historical abnormal developmental, medical, or psychiatric conditions. Mean IQ for the group was 116 (range, 101-135). None of the children were taking regular medication for any medical condition. These children were recruited as a pediatric control group for neuroimaging studies in children with various disorders. Informed consent was obtained from all subjects' parents or legal guardians. Children older than 13 years of age signed an assent form, whereas younger children gave verbal consent to the study. The study was approved by the Institutional Review Board at Wayne State University (Detroit, Michigan).

\section{Image Acquisition Protocol}

MR imaging scans were performed on a 3T Signa scanner (GE Healthcare, Milwaukee, Wisconsin) by using an 8-channel head coil. A 3D structural MR imaging scan, acquired by using a T1-weighted inversion recovery FSPGR sequence, and DTI ASSET data were obtained from each subject. The FSPGR images of the whole brain were acquired by using the following parameters: TR/TE/TI, 9.12/3.66/400 ms; flip angle, $13^{\circ}$; 120 axial sections, section thickness, $1.2 \mathrm{~mm}$; matrix size, $256 \times 256 \mathrm{~mm}$; and planar resolution, $0.94 \times 0.94 \mathrm{~mm}^{2}$. Moreover, whole brain DTI data were obtained by using a spin-echo echo-planar diffusion-weighted sequence in the axial plane applying 55 isotropic gradient directions with $b=1000 \mathrm{~s} / \mathrm{mm}^{2}$ and $1 \mathrm{~T} 2-$ weighted reference scan (B0). The DTI sequence consisted of the following imaging parameters: minimum 36 contiguous axial sections of $3 \mathrm{~mm}$ in thickness: TR/TE, $12,500 / 88.7 \mathrm{~ms}$; FOV, $240 \times 240 \mathrm{~mm}^{2}$; acquisition matrix size, $128 \times 128$ reconstructed to $256 \times 256$ with homodyne; and nominal in-plane resolution, $1.86 \times 1.86 \mathrm{~mm}^{2}$. The approximate scanning time for the DTI acquisition was 13 minutes. Double radio-frequency refocusing pulses and parallel imaging capability (ASSET factor of 2) were used to reduce eddy current and geometric distortion artifacts derived from echo-planar imaging. The children were not sedated, but their movements were carefully monitored during the MR imaging. None of the subject scans had apparent movement or other significant artifacts.

\section{Fiber Tracking Procedure}

Probabilistic fiber tracking was performed by using a Bayesian framework developed by Friman et al. ${ }^{22}$ The fiber tracking procedure and probability score calculation between seed and target regions have been described previously. ${ }^{23}$ In this model, the local PDF is determined at each step location based on the PDF of the neighboring 8 image voxels, weighted by the distance from the current position. The so-obtained local PDF is subsequently multiplied with a prior distribution, giving preference for continuation in the previous-step direction (zero probability for turns $>90^{\circ}$ ), and evaluated at 2562 predefined unit length vectors obtained by a 4 -fold tesselation of an icosahedron. Starting from a seed point, random samples are drawn from the posterior distribution, and fiber paths are created, which terminate when the local FA decreases below a preselected value $(0.20$ in this study). It has to be noted that this model assumes only 1 fiber direction in each voxel (single-fiber model), and any deviation from this model (such as fiber crossing or convergence) is translated into uncertainty in the posterior distribution. This uncertainty leads then locally to an increased probability of randomly sampled fibers to diverge into multiple directions, thus allowing a certain portion of fiber paths to cross low-anisotropy areas, hence realizing minor fiber tracts.

To quantitatively assess connectivity between a given source and target region, the average probability $p_{i}$ (equation 1 ) was calculated for each individual fiber path $i$ of length $N_{i}$ connecting the source and target region, where $p_{i j}$ is the randomly sampled probability of voxel $j$ $\left(j=1, \ldots N_{i}\right)$ determined from the local PDF calculated earlier. It has to be noted that in this context the index $j$ refers to voxels along path $i$ and not to the step length used to sample from the local PDF, which is smaller. The obtained value of $p_{i}$ can be interpreted as the average probability along the path with index $i$, independently of the path length. Because the number of fiber paths that connect a seed and target region is only a subset of all created fibers $(M \gg N)$, all path probabilities $p_{i}$ were subsequently normalized to the sampling space (total number of fiber paths $k=1, \ldots, M$ ) as $p_{i}^{\prime}$ (equation 2). Finally, the normalized probability score of connection between 2 regions $\mathrm{A}$ and B (termed $P_{A \rightarrow B}$ ) was calculated as the sum of $p_{i}{ }^{\prime}$ values from all fiber paths $l(l=1, \ldots L<M)$ connecting the source and target regions (equation 3).

$$
\begin{gathered}
p_{i}=\sqrt[N_{i}]{\prod_{j=1}^{N_{j}} p_{i j}} \\
p_{i}^{\prime}=\frac{p_{i}}{\sum_{k=1}^{M}} \\
p_{k} \quad P_{A \rightarrow B}=\sum_{l=1}^{L} p_{i}^{\prime}
\end{gathered}
$$

\section{Definition of Thalamic Seed Regions}

Bilateral thalami were manually outlined in each subject's native space by the same investigator (B.A.) to obtain seed regions for fiber tracking (Fig 1A). To facilitate the consistent demarcation of ROIs across the subjects and to correct for different head positions inside the scanner, we used an intersubject coregistration approach by using the SPM8 software package (www.fil.ion.ucl.ac.uk/spm). ${ }^{24}$ As a reference, we selected the $\mathrm{B} 0$ image of one of the subjects whose head was properly positioned to show bilateral thalami symmetrically in each 

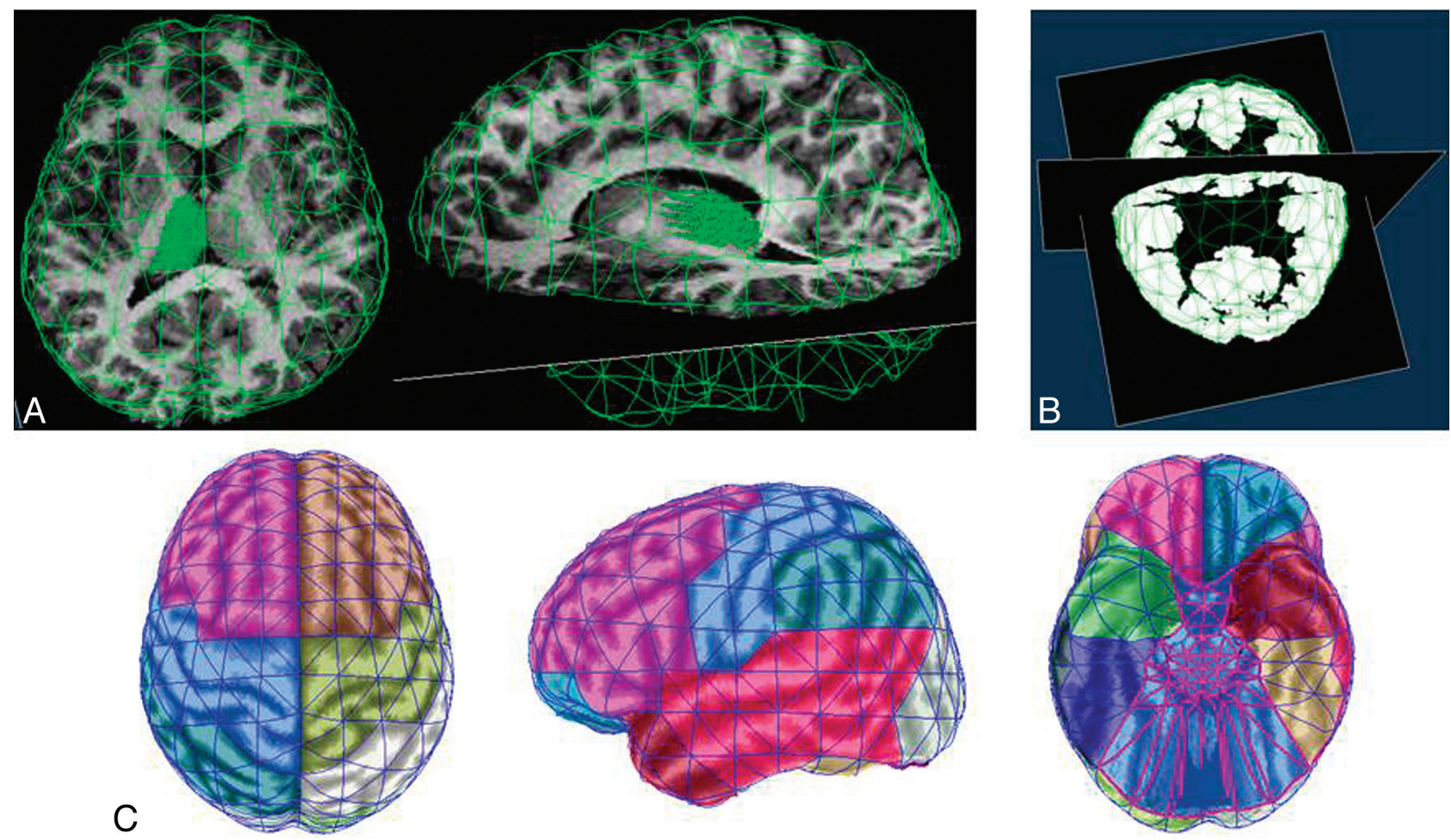

Fig 1. Images showing the seed and target definition for fiber tracking. $A$, Regions of interest were drawn on 7 consecutive planes of each subject's FA images to outline the thalamus. These representative images of 1 subject show the 3D seed region of the left thalamus loaded in the native FSPGR space. $B$, Representative image of the target layer (white color) in one of the subjects. The target mask contained the layer between the cortical surface and the 2-mm shrunken white matter mask. FTEs were obtained by extending the surface elements $30 \mathrm{~mm}$ deeper into the brain to select the underlying portion of the target mask. $C$, Superior, lateral, and inferior views of the ICBM152 template brain surface with the 8 cortical target regions on each side encompassing sets of surface elements. The cortical regions were defined by using main sulci as references. Those surface elements that extended a landmark sulcus were assigned to the region to which their largest part belonged. Importantly, the territory outlined with magenta (on the inferior surface) was not analyzed.

image plane. This B0 image was used as a target image for the B0 images of different subjects and a $12 d f$ affine transformation was applied for intersubject registration. The resulting transformation matrix also was applied to register the subjects' diffusion-weighted images into the reference space. Because the directions of the 3 gradient axes in the registered gradient data were rotated according to the applied transformation, we corrected the original gradient tables by multiplying the transformation matrix. The corrected gradient table was used for tractography analysis with the resampled gradient data.

The bilateral thalami were defined in 7 consecutive FA image planes with the medial border formed by the third ventricle, the posterior limb of the internal capsule as the lateral border, and the subthalamic nuclei and geniculate bodies as the inferior-posterior border (Fig 1A). To avoid the inclusion of voxels potentially belonging to adjacent structures with high FA values (eg, internal capsule, geniculate bodies), an upper FA threshold of 0.5 was used for fiber origination.

\section{Definition of Cortical Target Regions}

To define cortical target regions in native space that correspond spatially across subjects, we applied landmark-constrained conformal mapping of the cortical surface. ${ }^{25,26}$ Initially, to create a $3 D$ rendering of the cerebral hemispheres, all extracerebral structures (including cerebellum) were removed from the FSPGR image volumes by using BrainSuite2, ${ }^{27}$ and the resulting cortical surface was subsequently smoothed by using alpha shapes. ${ }^{28}$ Thus, the boundaries between white and gray matter and cortical sulci were exposed and projected onto the original cortical mask. Subsequently, parcellation of the cerebral cortex into 512 surface elements was achieved based on a set of manually defined major cortical landmarks (eg, central sulcus, Sylvian fissure). After conformal mapping and alignment of landmarks with corresponding landmarks of a reference brain (ICBM152 brain template; International Consortium on Brain Mapping) in canonical space, this procedure yielded homotopic surface elements in each subject's native space. ${ }^{25,26}$ Notably, the reference brain was not used as a template for spatial transformation and coregistration with individual images of the subjects, but its readily identifiable cortical landmarks served as a reference to yield surface elements with the same spatial relationship to landmarks in each subject's native space. To extend these surface elements inside the brain, thus creating FTEs, white matter voxels were initially extracted from FSPGR images by using the SPM8 software package. ${ }^{29}$ A shrink operation was then applied to the binarized white matter mask resulting in a white matter volume where the outer 2 -mm layer was removed. FTEs were then created by extending the surface elements $30 \mathrm{~mm}$ inside the brain to select the layer between the shrunken white matter and the cortical surface; thus, FTEs included gray matter and a thin layer of subcortical white matter (Fig 1B). This target definition method seemed to be well suited to assess fibers that reach the gray matter but may not be counted in case of potential subtle gray matter segmentation errors.

Subsequently, sets of surface elements representing 8 cortical regions: the sensorimotor, prefrontal, orbitofrontal, lateral temporal, inferior temporal, temporopolar, parietal, and occipital cortical regions were defined in both hemispheres based on their spatial proximity to major cortical landmarks (Fig $1 C$ ). In addition, regions representing the ipsilateral cortex were defined as the sum of all analyzed cortical regions in each hemisphere. Target regions were defined on the ICBM152 reference brain because its pattern of major landmarks 
represents population-average. However, the spatial accuracy of region definition was limited by the size (on average, $\sim 1.3 \mathrm{~cm}^{2}$ ) and shape (triangle) of the generated surface elements. Importantly, corresponding, actual target layers in the native DTI space of each subject were obtained by applying the transformation matrix between the DTI and FSPGR space. This transformation matrix was calculated by coregistering the B0 image volumes (extracted from the reoriented DTI data) and the FSPGR volumes by using a well-established 3D registration technique (VINCI $2.50 .0 ;^{30}$ ).

\section{Data Analysis}

To quantify the connectivity between the source region (left and right thalamus) and the predefined cortical target regions, initially a probability score of connection (ie, connectivity score) between the thalamus and each ipsilateral FTE was calculated. Subsequently, connectivity scores for all predefined cortical target regions were calculated by summing the connectivity scores of the FTEs comprising each target. Importantly, fiber paths crossing the midline were not analyzed.

Initially, we set out to reproduce a well-known symmetrical pattern of the corticospinal tract to validate that the applied methodology is capable of measuring known symmetries of the brain, and therefore, identified asymmetries are likely real. The posterior limb of the internal capsule was used as a seed and the ipsilateral sensorimotor cortical regions as targets on each side. Probability scores of the left and right sensorimotor areas were compared by using a paired $t$ test.

To calculate interrater reliability for connectivity measures for thalamocortical connections, seed ROIs were redrawn by another investigator (blinded to the original ROIs) and the fiber tracking was repeated in 5 randomly selected subjects. Initially, a ratio between the intersection and union of the volumes of corresponding ROIs (A and B) defined by the 2 observers was calculated. Agreement between 2 observers with respect to connectivity scores for the 8 cortical regions was assessed by using the ICC test. In addition, the reproducibility of hemispheric differences was tested by applying the same method to regional hemispheric AIs (\%); calculated as $200 \times(\mathrm{L}-\mathrm{R}) /(\mathrm{L}+\mathrm{R})$, where $\mathrm{L}$ represents connectivity scores for left, and $\mathrm{R}$ represents those for right hemispheric homotopic regions)]. Furthermore, the fiber tracking and connectivity score calculation of thalamocortical fibers were repeated in 5 randomly selected subjects with a modified set of parameters (FTE thickness, $25 \mathrm{~mm}$; upper FA threshold of seed region, 0.55; lower FA threshold for fiber termination, 0.15).

To demonstrate the average $3 \mathrm{D}$ brain surface map of thalamocortical fiber connections in the analyzed age group, connectivity scores for each homotopic FTE in every subject were averaged, and the surface elements on the ICBM152 template brain surface were color-coded based on their calculated average connectivity to the ipsilateral thalamus.

Because the necessary statistical assumptions were not violated (normality of side-differences was investigated with the KolmogorovSmirnov test), connectivity scores for each region were compared by using separate paired samples $t$ tests to determine whether there was a difference between homotopic cortical regions in the 2 hemispheres with respect to their connectivity to the ipsilateral thalamus. In addition, FA of fibers showing robust, reproducible hemispheric asymmetry was measured as follows. The mean FA of left-sided fibers was compared with that of the contralateral homotopic white matter area (left-sided fibers were mirrored along the midline) by using a paired $t$ test.

The potential association between thalamic connectivity of cortical regions and age was tested by using the Pearson product moment correlations. To test potential age-related changes of connectivity asymmetries, AIs (calculated as indicated above) for each cortical target region were correlated with age by using the Pearson correlation. To control for type I errors associated with multiple comparisons, the pair-wise $\alpha$ was adjusted to $P=.01$. The statistical analysis was performed by using SPSS 17.0 (SPSS, Chicago, Illinois).

\section{Results}

\section{Reliability}

The ratio between the intersection and union of ROI volumes [ie, $(\mathrm{A} \cap \mathrm{B}) /(\mathrm{A} U \mathrm{~B})]$ defined by the 2 observers ranged between $72.4 \%$ and $89.4 \%$ (mean, $78.5 \%$ ). The ICC for regional connectivity scores ranged between 0.989 and $0.999(P<.001)$ and that for the asymmetries was in the range of $0.980-0.997$ $(P<.001)$, showing excellent reproducibility of the results. No significant side difference in the connectivity between the posterior limb of the internal capsule and the sensorimotor cortex was determined (left, $29.5 \pm 5 \%$ vs right, $27.9 \pm 6 \%$; $P=.48)$.

\section{Thalamocortical Connectivity Map}

The average cortical connectivity map (Fig 2) demonstrates the spatial distribution of thalamocortical fibers with the strongest thalamic connection to the sensorimotor cortex. The average map also illustrates that certain cortical regions show considerable hemispheric differences in their thalamic connectivity (eg, prefrontal, orbitofrontal cortex).

\section{Hemispheric Asymmetries in Thalamocortical Connectivity}

The left cerebral cortex had significantly higher overall connectivity scores than the right cerebral cortex, indicating stronger thalamocortical connectivity on the left side $(P<$ .001 ; Fig 3). Regional analysis of asymmetries in thalamocortical connectivities showed that the prefrontal, orbitofrontal, as well as inferior temporal cortex had significantly stronger thalamic connections on the left side than on the right side $(P<.001, P=.004$, and $P=.003$, respectively $)$. The left $>$ right asymmetry was the most robust in the prefrontal area, with all 15 subjects having higher connectivity scores in the left hemisphere. In addition, the lateral temporal region showed a trend for a left $>$ right asymmetry $(P=.029)$. Conversely, we found a trend for a right $>$ left asymmetry for connectivity scores in the sensorimotor and parietal regions $(P=$ .057 and $P=.02$, respectively). Finally, FA values derived from left thalamo-prefrontal fibers were higher than those of the homotopic right hemispheric white matter area $(0.44 \pm 0.025$ vs $0.42 \pm 0.036 ; P=.03)$. Importantly, FA values were closely associated with the related connectivity scores (left: $r=0.75$, $P=.001$; right: $r=0.7, P=.004$ ).

The reanalysis of a random subset of the sample $(n=5)$ with different predefined parameters showed that the regional connectivity scores were well reproducible (ICC, 0.9000.993; $P<.001)$. The reanalysis could reproduce the robust left $>$ right asymmetry in the overall thalamocortical as well as thalamo-prefrontal connectivity (all 5 reanalyzed subjects showed again left $>$ right laterality). However, side differences of regions with low thalamic connectivity (ie, orbitofrontal, inferior temporal) were parameter-dependent (laterality of AI 
top

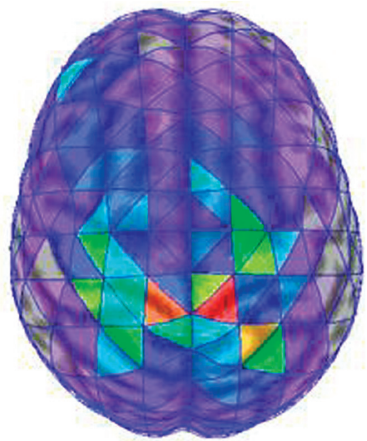

bottom

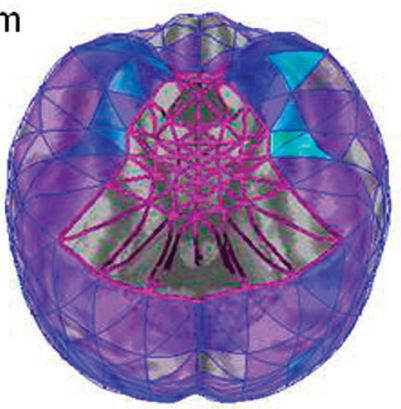

left

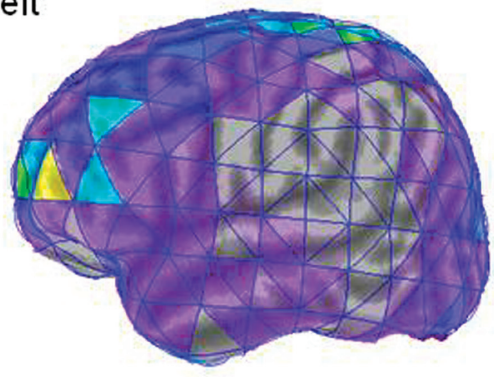

right

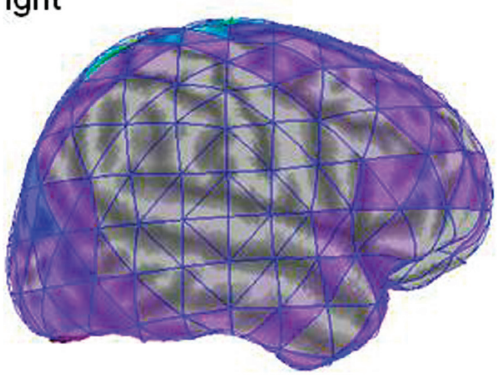

front

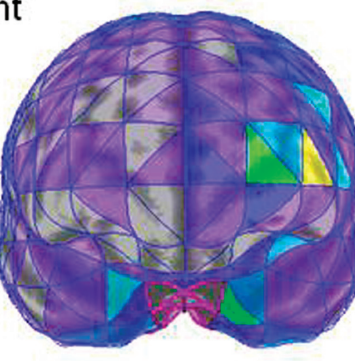

back

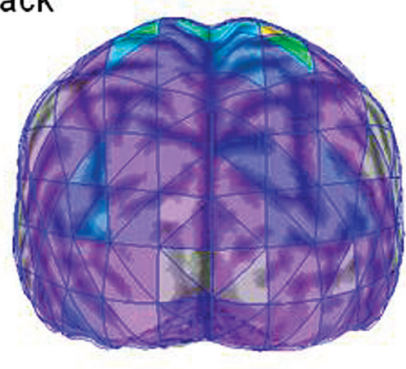

Fig 2. Average thalamocortical connectivity map of the 15 subjects visualized on the $3 \mathrm{D}$ brain surface of the ICBM152 template brain. The thalamocortical connectivity scores of each homotopic surface element were averaged among the 15 subjects, and the surface elements of the template brain were color-coded based on these average values. Notably, only the ipsilateral thalamic connections of each hemisphere are shown. It can be appreciated that the bilateral sensorimotor cortical areas have the strongest thalamic connectivity and that certain cortical regions (eg, prefrontal, inferior temporal) show profound hemispheric asymmetries.

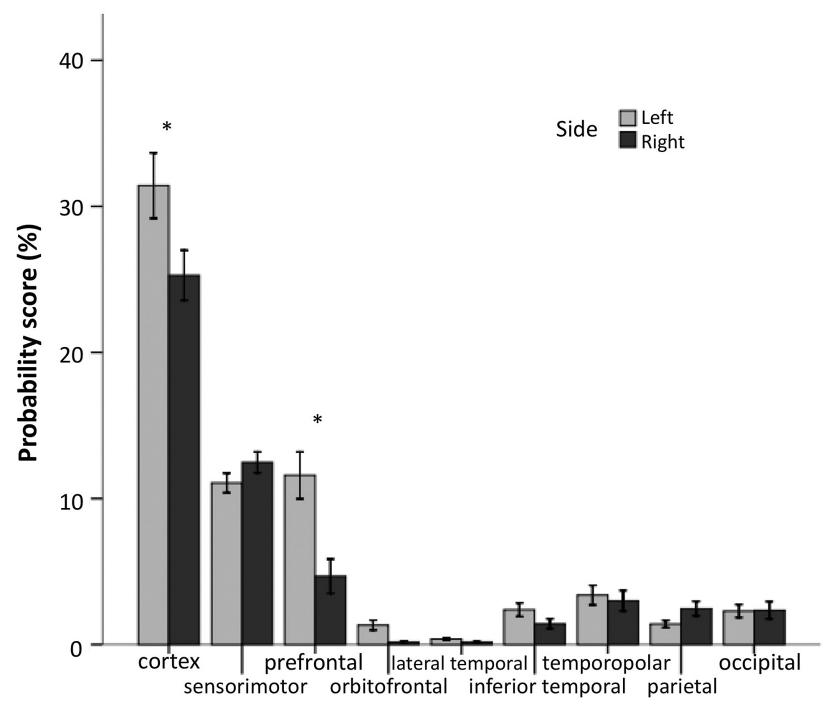

Regions

Fig 3. Bar graph showing the thalamocortical connectivity scores (mean $\pm S E$ ) for the cortex (sum of the analyzed 8 regions) and each individual cortical region. Significant $(P<$ .01) and reproducible (parameter-independent) left $>$ right hemispheric asymmetries are indicated with asterisk.

switched in 3/5 for inferior temporal and 2/5 cases for orbitofrontal region).

\section{Age-Related Changes in Thalamocortical Connectivity}

The overall thalamocortical connectivities on both sides showed a trend for higher scores in older children (left: $r=$ $0.63, P=.01$; right: $r=0.55, P=.035)$. The analysis of regional connectivity scores showed a significant positive correlation with age in the prefrontal cortex on both sides (left: $r=$

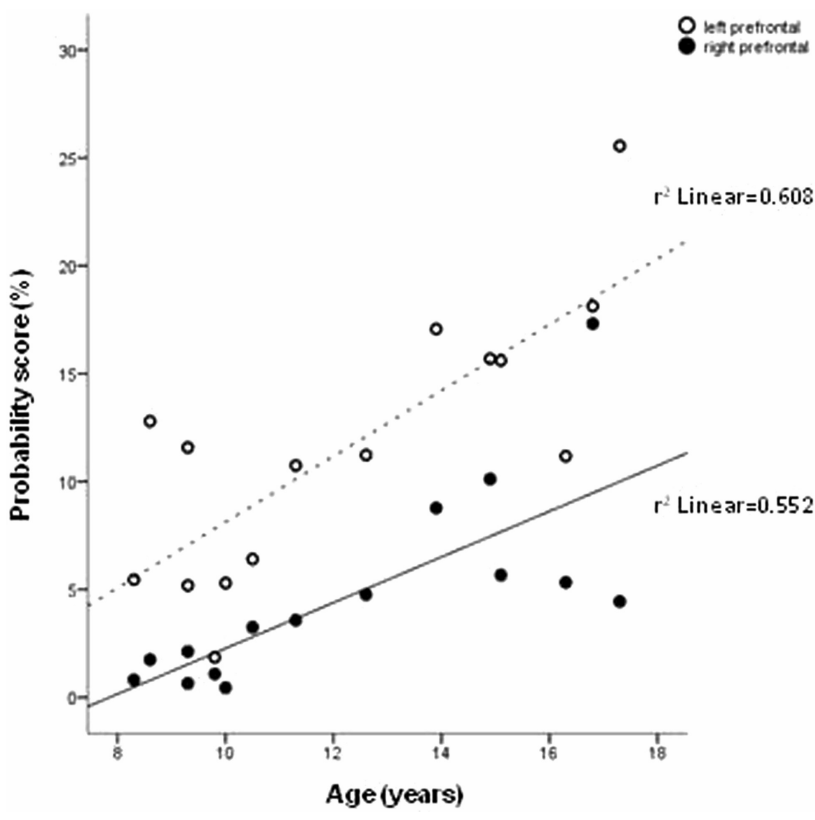

Fig 4. Scatterplot showing a significant positive correlation between age and thalamic connectivity scores of the left and right prefrontal cortex $(P=.001$ and $P=.002$, respectively).

$0.78, P=.001$; right: $r=0.74, P=.002$ ) (Fig 4). In addition, connectivity scores of the left occipital and the right orbitofrontal regions showed a trend for positive correlation with age ( $r=0.55, P=.036$ and $r=0.60, P=.018$, respectively). In contrast, an inverse association with age was found in the left inferior temporal $(r=-0.65, P=.009)$ and right sensorimotor $(r=-0.59, P=.02)$ cortex.

There were no significant correlations between AIs of re- 
gional thalamocortical connectivities and age; however, the asymmetry of the thalamic connectivity of the prefrontal cortex showed a trend for decrease with age $(r=-0.52, P=$ .046), suggesting a slight decrease in the left $>$ right asymmetry in this region.

\section{Discussion}

The present study aimed to quantitatively demonstrate the normal cortical pattern and variances of thalamocortical fiber connections in children and adolescents by using probabilistic tractography and cortical mapping. Our main finding is the robust hemispheric asymmetry in thalamocortical connectivity, which is mainly driven by the markedly stronger thalamic connection of the left frontal cortex compared with the right frontal cortex. Moreover, we demonstrated developmental (age-related) changes in the overall as well as regional thalamocortical connections. Particularly, the bilateral thalamic connectivity to the prefrontal cortex showed a marked age-related increase, whereas the thalamic connectivity of the left inferior temporal and right sensorimotor regions decreased. These changes probably reflect a complex, regionspecific developmental process and dynamic remodeling of thalamocortical circuitries that continues throughout adolescence and perhaps beyond.

\section{Hemispheric Asymmetries in Thalamocortical Connectivity}

Development of structural asymmetries is thought to be associated with the normal hemispheric lateralization of cognitive functions. ${ }^{12,13}$ Particularly, white matter tracts projecting to the temporal lobes (arcuate fasciculus, uncinate fasciculus, cingulum, inferior longitudinal fasciculus) are characterized by left predominance in parameters such as volume and FA. ${ }^{31-33}$ However, projection pathways such as the corticospinal tract tend to be symmetric. ${ }^{34}$ Interestingly, a certain coupling exists between white matter pathways with respect to their microstructure that may reflect phylogenetic similarities, functional similarities, or both between tracts. ${ }^{35}$ Indeed, increasing evidence supports the notion that asymmetries in the adult brain are the result of a long developmental process. ${ }^{36} \mathrm{~A}$ pediatric DTI study using FA and ADC as measures of white matter integrity showed left greater than right FA values in the anterior limb of the internal capsule and centrum semiovale. ${ }^{37}$ Bonekamp et $\mathrm{al}^{38}$ reported significant asymmetries for ADC and FA, with higher FA and/or lower ADC values in the left frontal white matter, genu of corpus callosum, anterior white matter, superior fronto-occipital fasciculus, superior corona radiata, centrum semiovale, and cingulum. In addition, Wilde et $\mathrm{al}^{9}$ found lower ADC values in the left temporal, prefrontal, anterior internal capsular, and thalamic regions and higher FA values on the left side in the internal capsule, cingulate, and thalamus compared with the right side.

To our knowledge, the present work is the first study to quantify the asymmetries and age-related changes of regional cortical distribution of thalamocortical connectivities in healthy children by using DTI-based probabilistic tractography. Our results of higher thalamocortical connectivity on the left side are generally consistent with previous data that showed greater anisotropy in the left hemisphere compared with the right in the thalamus and in white matter regions of putative thalamocortical pathways. ${ }^{9,37,38}$ The significantly stronger thalamic connections of the left prefrontal cortex compared with the right homotopic areas are also in line with earlier studies of similar age groups reporting left $>$ right FA, right $>$ left ADC asymmetries, or both in frontal white matter regions.9,37,38 The general agreement between pediatric and adult studies with respect to the left $>$ right asymmetry of FA is that these microstructural asymmetries begin in childhood. ${ }^{39}$ Notably, the previously detected hemispheric asymmetries in measures of white matter integrity (FA asymmetry up to $28 \%)^{9}$ were small as opposed to our observations of marked side differences (up to 10-fold asymmetry in thalamo-prefrontal connectivity). This is probably attributable to the different methodology and the fact that connectivity scores are not a mere reflection of local FA and ADC values.

Some previous observations may provide clues for understanding the mechanisms of asymmetries of thalamocortical connectivity. A possible explanation for the left>right asymmetry of thalamo-prefrontal connectivity in children could be the different timing of gray matter maturation in the 2 hemispheres. This was demonstrated in a longitudinal study showing that the left prefrontal cortex matures earlier than the right in a sample of predominantly right-handed children. ${ }^{15}$ In addition, because structural asymmetry correlates of left-lateralized language specialization have been found in right-handed subjects, ${ }^{38,40}$ other functional hemispheric specializations (eg, working memory, quantitative skills) also may be implicated in asymmetries of thalamocortical connections. ${ }^{9,41}$ However, larger studies with neurobehavioral correlations are needed to elucidate the functional correlates and detailed developmental trajectories of asymmetries in thalamocortical fiber distribution.

\section{Age-Related Changes in Thalamocortical Connectivity}

Parallel to the dynamic behavioral, cognitive, and emotional development during childhood and adolescence, significant structural changes occur in the maturing brain. This process involves both the gray matter and the white matter. ${ }^{36}$ Numerous developmental studies of children, adolescents, and young adults have demonstrated widespread age-associated FA increases and ADC decreases involving the bulk of white matter tracts, including thalamocortical pathways and deep gray matter structures. ${ }^{9,14,16-18,37,38,40,42,43}$ Although the exact physiologic background of these age-related diffusion changes is unclear, some of their cognitive and behavioral correlates have been described previously. ${ }^{41,44}$

Developmental changes of thalamocortical connections have not been specifically investigated previously. However, the age-associated increase in the overall thalamocortical as well as thalamo-prefrontal connectivity demonstrated in the present study is consistent with results of earlier reports showing increasing FA with age in areas corresponding to thalamocortical tracts and the anterior limb of the internal capsule. ${ }^{16,37,38,42}$ In addition, it has been shown that synaptic pruning in the prefrontal cortex continues into middle adolescence. ${ }^{45}$ The prefrontal cortex and the basal ganglia-thalamus-frontal cortex circuitry have been implicated in "highorder" cognitive functions such as working memory, learning, attention, and behavioral functions. ${ }^{3,41,46}$ The age-related gray matter loss, which extends to the dorsolateral prefrontal cor- 
tex in adolescence, and the increasing myelination of the prefrontal subcortical white matter along with the basal ganglia and thalami have been suggested as underlying substrates of the development of complex cognitive functioning. ${ }^{15,16}$ In line with the notion that the prefrontal cortex develops relatively late,${ }^{15}$ here we show that the thalamic connectivity of this particular cortical region undergoes a remarkable age-related increase during late childhood and adolescence. This may be one of the neurologic substrates of maturation of high-order executive functions.

In addition to age-related increases, we also have observed age-related decreases in the thalamic connectivity of the left inferior temporal and right sensorimotor cortex. This may suggest a dynamic, region-specific remodeling of thalamocortical pathways. In our study, asymmetries in regional thalamocortical connectivities did not correlate with age, except for a trend for decreasing left>right AI of the thalamo-prefrontal connectivity. Interestingly, Wilde et $\mathrm{al}^{9}$ recently described decreasing left > right asymmetry of frontal FA in a similar study population. Our limited age range precluded the study of maturational changes in thalamocortical connectivity in young adults, and it remains unclear whether the observed asymmetries diminish in early adulthood.

The fronto-(striato)-thalamic pathways are clinically relevant as they have been implicated in several developmental disorders (eg, attention deficit hyperactivity disorder, obsessive-compulsive disorder), often manifesting with impairments in working memory, attention, or inhibition. ${ }^{11,47}$ Thus, we speculate that the development of thalamo-prefrontal pathways may be altered in some of these disorders. Future studies could build upon the current data to elucidate the role of abnormal development of thalamo-cortical connections in these conditions.

\section{Study Limitations}

The present study has a cross-sectional design with limited number of subjects, limited age range, and small number of girls, which precludes analysis of sex effects. A longitudinal DTI study with a larger sample size and wider age range could elucidate the distinct developmental trajectories of cortical regions regarding their thalamic connections with a better power. Furthermore, our intriguing, however preliminary results need to be verified with approaches implementing diffusion spectrum imaging or high angular resolution diffusion imaging. ${ }^{48}$ Importantly, we did not analyze the potential cognitive correlates of the strength and side differences of thalamocortical connections; such an analysis would require a larger sample with a wider cognitive range to yield definitive conclusions.

Indeed, our methodology is novel and the results require further verification with other methods. However, the main finding of the study was robust and well reproducible, and side differences of thalamo-prefrontal connections were further supported by left $>$ right FA values of related white matter areas. The limitation regarding our cortical mapping technique is that we excluded from the analysis those, relatively small, brain surface areas (representing some inferio-medio-temporal, inferio-occipital regions) that have the highest potential for distortions in canonical space, resulting in compromised spatial accuracy of the generated surface elements. Similarly, the current approach is not applicable for the quantitative analysis of fibers terminating in some medial cortical regions (eg, cingulate cortex). Notably, despite the advantages of the probabilistic approach, fibers with high degree inflections or minor pathways crossing major fibers may not be detected. This probably explains the low or zero connectivity scores of FTEs on the lateral sensorimotor areas. Also, the current approach underestimates the effect of crossing white matter tracts within a voxel; therefore, it cannot be ruled out that the observed asymmetries and age-related changes in thalamocortical fibers reflect the differences in the microstructural architecture of these regions. Importantly, the relatively wide range of regional connectivity scores (as opposed to earlier works showing small variations in FA) among our subjects may indicate real variations in the pattern of thalamocortical pathways in children. The behavioral and cognitive correlates of these interindividual variances remain unclear.

\section{Conclusions}

Our results uncover intriguing, though preliminary information about hemispheric differences in thalamocortical connections in the maturing brain. The present data suggest that the dominant (left) hemisphere has stronger thalamocortical connections in healthy children $\geq 8$ years of age and that this asymmetry is mainly driven by the significantly higher thalamo-prefrontal connectivity in the left hemisphere. The overall thalamocortical connectivity increases bilaterally with age within the studied age range with the most robust increase in the prefrontal regions. However, thalamic connectivity of some cortical regions decreases, probably suggesting a continuous, dynamic remodeling of thalamocortical pathways during maturation. The described normal asymmetries and agerelated changes of thalamocortical fiber distribution may be helpful to understand abnormal thalamocortical connections in certain developmental disorders.

\section{Acknowledgments}

We gratefully acknowledge the help of Piti Sinsoongsud in the image preprocessing.

\section{References}

1. Haber SN, Calzavara R. The cortico-basal ganglia integrative network: the role of the thalamus. Brain Res Bull 2009;78:69-74

2. Sarnthein J, Morel A, von Stein A, et al. Thalamocortical theta coherence in neurological patients at rest and during a working memory task. Int J Psychophysiol 2005;57:87-96

3. Cummings JL. Frontal-subcortical circuits and human behavior. Arch Neurol 1993;50:873-80

4. Tekin S, Cummings JL. Frontal-subcortical neuronal circuits and clinical neuropsychiatry: an update. J Psychosom Res 2002;53:647-54

5. Zhang D, Snyder AZ, Shimony JS, et al. Noninvasive functional and structural connectivity mapping of the human thalamocortical system. Cereb Cortex 2010;20:1187-94

6. Rosanova M, Casali A, Bellina V, et al. Natural frequencies of human corticothalamic circuits. J Neurosci 2009;29:7679-85

7. Jones EG. The thalamus of primates. In: Bloom FE, Bjorklund A, Hokfelt T, eds. The Primate Nervous System, Part II. Amsterdam, the Netherlands: Elsevier Science;1998:1-298

8. Johansen-Berg H, Behrens TE, Sillery E, et al. Functional-anatomical validation and individual variation of diffusion tractography-based segmentation of the human thalamus. Cereb Cortex 2005;15:31-39

9. Wilde EA, McCauley SR, Chu Z, et al. Diffusion tensor imaging of hemispheric asymmetries in the developing brain. J Clin Exp Neuropsychol 2009;31:205-18

10. Lüders E, Narr KL, Thompson PM, et al. Hemispheric asymmetries in cortical thickness. Cereb Cortex 2006;16:1232-38 
11. Shaw P, Lalonde F, Lepage C, et al. Development of cortical asymmetry in typically developing children and its disruption in attention-deficit/hyperactivity disorder. Arch Gen Psychiatry 2009;66:888-96

12. Toga AW, Thompson PM. Mapping brain asymmetry. Nat Rev Neurosci 2003;4:37-48

13. Galaburda AM, LeMay M, Kemper TL, et al. Right-left asymmetrics in the brain. Science 1978;199:852-56

14. Mukherjee P, Miller JH, Shimony JS, et al. Diffusion-tensor MR imaging of gray and white matter development during normal human brain maturation. AJNR Am J Neuroradiol 2002;23:1445-56

15. Gogtay N, Giedd JN, Lusk L, et al. Dynamic mapping of human cortical development during childhood through early adulthood. Proc Natl Acad Sci U S A 2004;101:8174-79

16. Barnea-Goraly N, Menon V, Eckert M, et al. White matter development during childhood and adolescence: a cross-sectional diffusion tensor imaging study. Cereb Cortex 2005; 15:1848-54

17. Giorgio A, Watkins KE, Chadwick $M$, et al. Longitudinal changes in grey and white matter during adolescence. Neuroimage 2009;49:94-103

18. Lebel C, Walker L, Leemans A, et al. Microstructural maturation of the human brain from childhood to adulthood. Neuroimage 2008;40:1044-55

19. Behrens TE, Woolrich MW, Jenkinson M, et al. Characterization and propagation of uncertainty in diffusion-weighted MR imaging. Magn Reson Med 2003;50:1077-88

20. Wechsler D. Wechsler Preschool and Primary Scale of Intelligence, 3rd ed. San Antonio, Texas: The Psychological Corporation; 2002

21. Reynolds CR, Kamphaus RW. Behavioral Assessment System for Children, 2nd ed. Circle Pines, Minnesota: American Guidance Service; 2004

22. Friman O, Farneback G, Westin CF. A Bayesian approach for stochastic white matter tractography. IEEE Trans Med Imaging 2006;25:965-78

23. Behen ME, Muzik O, Saporta AS, et al. Abnormal fronto-striatal connectivity in children with histories of early deprivation: a diffusion tensor imaging study. Brain Imaging Behav 2009;3:292-97

24. Ashburner J, Andersson JL, Friston KJ. High-dimensional image registration using symmetric priors. Neuroimage 1999;9:619-28

25. Muzik O, Chugani DC, Zou G, et al. Multimodality data integration in epilepsy. Int J Biomed Imaging 2007;2007:13963

26. Alkonyi B, Juhasz C, Muzik O, et al. Quantitative brain surface mapping of an electrophysiologic/metabolic mismatch in human neocortical epilepsy. Epilepsy Res 2009;87:77-87

27. Shattuck DW, Leahy RM. BrainSuite: an automated cortical surface identification tool. Med Image Anal 2002;6:129-42

28. Edelsbrunner H, Mucke EP. Three-dimensional alpha shapes. ACM ACM Trans Graph 1994;13:43-72

29. Ashburner J, Friston KJ. Unified segmentation. Neuroimage 2005;26:839-51

30. Cízek J, Herholz K, Vollmar S, et al. Fast and robust registration of PET and MR images of human brain. Neuroimage 2004;22:434-42

31. Gong G, Jiang T, Zhu C, et al. Side and handedness effects on the cingulum from diffusion tensor imaging. Neuroreport 2005;16:1701-05
32. Rodrigo S, Naggara O, Oppenheim C, et al. Human subinsular asymmetry studied by diffusion tensor imaging and fiber tracking. AJNR Am J Neuroradiol 2007;28:1526-31

33. Wakana S, Caprihan A, Panzenboeck MM, et al. Reproducibility of quantitative tractography methods applied to cerebral white matter. Neuroimage 2007;36:630-44

34. Reich DS, Smith SA, Jones CK, et al. Quantitative characterization of the corticospinal tract at 3T. AJNR Am J Neuroradiol 2006;27:2168-78

35. Wahl M, Li YO, Ng J, et al. Microstructural correlations of white matter tracts in the human brain. Neuroimage 2010;51:531-41

36. Reiss AL, Abrams MT, Singer HS, et al. Brain development, gender and IQ in children. A volumetric imaging study. Brain 1996;119(Pt 5):1763-74

37. Snook L, Paulson LA, Roy D, et al. Diffusion tensor imaging of neurodevelopment in children and young adults. Neuroimage 2005;26:1164-73

38. Bonekamp D, Nagae LM, Degaonkar M, et al. Diffusion tensor imaging in children and adolescents: reproducibility, hemispheric, and age-related differences. Neuroimage 2007;34:733-42

39. Powell HW, Parker GJ, Alexander DC, et al. Hemispheric asymmetries in language-related pathways: a combined functional MRI and tractography study. Neuroimage 2006;32:388-99

40. Verhoeven JS, Sage CA, Leemans A, et al. Construction of a stereotaxic DT atlas with full diffusion tensor information for studying white matter maturation from childhood to adolescence using tractography-based segmentations. Hum Brain Mapp 2010;31:470-86

41. Olesen PJ, Nagy Z, Westerberg H, et al. Combined analysis of DTI and fMRI data reveals a joint maturation of white and grey matter in a fronto-parietal network. Brain Res Cogn Brain Res 2003;18:48-57

42. Schmithorst VJ, Wilke M, Dardzinski BJ, et al. Correlation of white matter diffusivity and anisotropy with age during childhood and adolescence: a cross-sectional diffusion-tensor MR imaging study. Radiology 2002;222: 212-18

43. Tamnes CK, Ostby Y, Fjell AM, et al. Brain maturation in adolescence and young adulthood: regional age-related changes in cortical thickness and white matter volume and microstructure. Cereb Cortex 2010;20:534-48

44. Schmithorst VJ, Wilke M, Dardzinski BJ, et al. Cognitive functions correlate with white matter architecture in a normal pediatric population: a diffusion tensor MRI study. Hum Brain Mapp 2005;26:139-47

45. Huttenlocher PR, Dabholkar AS. Regional differences in synaptogenesis in human cerebral cortex. J Comp Neurol 1997;387:167-78

46. Kwon H, Reiss AL, Menon V. Neural basis of protracted developmental changes in visuo-spatial working memory. Proc Natl Acad Sci U S A 2002;99:13336-41

47. Huyser C, Veltman DJ, de Haan E, et al. Paediatric obsessive-compulsive disorder, a neurodevelopmental disorder? Evidence from neuroimaging. Neurosci Biobehav Rev 2009;33:818-30

48. Yeh FC, Wedeen VJ, Tseng WY. Generalized q-sampling imaging. IEEE Trans Med Imaging 2010;29:1626-35 